\title{
BMJ Open One-year visual outcome of small incision lenticule extraction (SMILE) surgery in high myopic eyes: retrospective cohort study
}

Wenjing Wu, Yan Wang, Hui Zhang, Jiamei Zhang, Hua Li, Rui Dou

To cite: Wu W, Wang Y, Zhang $\mathrm{H}$, et al. One-year visual outcome of small incision lenticule extraction (SMILE) surgery in high myopic eyes: retrospective cohort study. BMJ Open 2016;6:e010993.

doi:10.1136/bmjopen-2015010993

- Prepublication history for this paper is available online. To view these files please visit the journal online (http://dx.doi.org/10.1136/ bmjopen-2015-010993).

Received 28 December 2015 Revised 29 August 2016 Accepted 31 August 2016

CrossMark

Tianjin Ophthalmology and Visual Science Key Laboratory, Tianjin Eye Hospital \& Eye Institute, Clinical College of Ophthalmology, Tianjin Medical University, Tianjin, China

Correspondence to Professor Yan Wang; wangyan7143@vip.sina.com

\section{ABSTRACT}

Objective: To determine whether the long-term visual outcome of small incision lenticule extraction (SMILE) surgery is consistent with the short-term results in high myopic eyes.

Design: Retrospective cohort study; data collected from 8 August 2011 to 31 August 2015.

Setting: Single refractive surgery centre.

Participants: A total of 156 eyes were studied: 65 eyes of 39 subjects (22 female/17 male) in the high myopic group (manifest refraction spherical equivalent (MRSE) $\geq-6.0 \mathrm{D}$ ), and 91 eyes of 54 subjects (29 female/25 male) in the control group (MRSE $<-6.0 \mathrm{D})$. The inclusion criteria were subjects who had follow-ups after 1 day, 1 week, 1 month, 3 , 6 months and 1 year with the manifest refraction, uncorrected and corrected distance visual acuity (UDVA/CDVA). There were no statistically significant differences between the two groups in the subjects' gender, age, or cylindrical dioptre, preoperatively $(p=0.835, p=0.055, p=0.341$, respectively).

\section{Primary and secondary outcome measures:} UDVA, refractive stability, safety index (postoperative CDVA/preoperative CDVA), and predictability (the percentage of eyes within $\pm 0.50 \mathrm{D}$ ).

Results: In both groups, the 1-year UDVA and safety index were significantly better than results at 1 day (high myopic group: $p=0.035, p<0.001$; control group: $p<0.016, p<0.001$ ); the 1 -year predictability showed no significant difference with the short-term results ( $p=1.00$ in both groups). In the high myopic eyes, the 1-year MRSE was significantly worse than the short-term result $(p=0.048)$. To correct it, the added magnitude (D) for the high myopic eyes may equal $0.13 \times$ Attempted SE (D) $-0.66 \mathrm{D}$. However, the postoperative MRSE showed no differences from 1 day to 1 year $(p=0.612)$ in the control group.

Conclusions: The 1-year visual outcomes were better than the short-term results after the SMILE surgery on the visual acuity and safety. However, the high myopic eyes suffered a significant regression at 1 year, which may be corrected by adding additional magnitude to the SE for high myopic eyes.

\section{Strengths and limitations of this study}

- Although many previous studies have investigated the early visual and refractive outcomes of small incision lenticule extraction (SMILE), the long-term observations of the SMILE procedure is far from established. This is 1-year study assesses the visual recovery, refractive stability, safety and predictability.

- Moreover, the long-term result of the high myopic eyes, which is a major concern in the prognosis of the patient, has not been well studied. We compared the 1-year visual outcomes between high myopic eyes and low to moderate myopic eyes. We also assessed whether the long-term results are consistent with the short-term results in high myopic eyes.

- This study did not assess the corneal biomechanics, or the high order aberrations. In addition, this is a retrospective cohort study, potentially prone to selection bias and other confounding variables. Future randomised controlled studies with large samples are needed to confirm the authenticity of the results.

\section{INTRODUCTION}

Small incision lenticule extraction (SMILE) is a new keratorefractive procedure to correct myopia and astigmatism. ${ }^{2}$ The short-term clinical results have been quite intensively investigated in the past several years. ${ }^{1-10}$ A major concern of SMILE surgery today is the long-term visual outcome, especially in high myopic eyes. ${ }^{11}{ }^{12}$ Some studies have reported the 1-year observations of SMILE surgery; ${ }^{13-17}$ however, long-term studies of SMILE surgery in high myopic eyes are far from being established. It has not been determined whether the long-term visual outcomes are consistent with the short-term results in high myopic eyes after SMILE surgery. This may be important to answer patients' concerns about clinical regression following SMILE surgery. 
Hence, this study was performed to investigate the shortterm and long-term (1-year) visual outcomes of the SMILE procedure in high myopic eyes, comparing differences in refraction, visual recovery, safety and predictability.

\section{PATIENTS AND METHODS \\ Ethics statement}

This study was approved by the Institutional Review Board of Tianjin Eye Hospital and adhered to the tenets of the Declaration of Helsinki. Written informed consent was obtained from all the patients.

\section{Subjects}

This was a retrospective cohort study. We reviewed the clinical charts of the subjects who underwent SMILE surgery in the refractive surgery centre of Tianjin Eye Hospital from 8 August 2011 to 31 August 2015. The inclusion criteria were subjects who had follow-ups at 1 day, 1 week, 1 month, 3 months, 6 months and 1 year postoperatively together with the manifest refraction, and uncorrected and corrected distance visual acuity (UDVA/ CDVA) examinations. The study involved a total of 156 myopic eyes. They were divided into two groups according to their preoperative manifest refraction spherical equivalent (MRSE): 65 eyes of 39 subjects (22 female/17 male) in the high myopic group (MRSE $\geq-6.0 \mathrm{D}$ ), and 91 eyes of 54 subjects ( 29 female/25 male) in the control group (MRSE $<-6.0 \mathrm{D}$ ). Theoretically, it is better to compare the visual outcomes among low myopic eyes, moderate myopic eyes and high myopic eyes. But clinically, most of the subjects who underwent corneal refractive surgery have had high myopia or moderate myopia. Only a few eyes with a low degree of myopia had the surgery. So we put the low myopia and moderate myopia eyes together as the control group. In the high myopia group, the mean $\pm \mathrm{SD}$ patient age was $23.68 \pm 5.25$ years, ranging from 18 to 39 years. The preoperative MRSE was $-6.90 \pm 0.86 \mathrm{D}$, ranging from -6.0 to $-9.50 \mathrm{D}$; the sphere was $-6.49 \pm 0.93 \mathrm{D}$, ranging from -4.75 to $-9.25 \mathrm{D}$; and the cylinder was $-0.82 \pm 0.68 \mathrm{D}$, ranging from 0.00 to $-3.50 \mathrm{D}$. In the control group, the mean patient age was $22.18 \pm 4.41$ years, ranging from 18 to 38 years. The preoperative MRSE were $-4.69 \pm 0.96 \mathrm{D}$, ranging from -1.25 to $-5.875 \mathrm{D}$; the sphere was $-4.21 \pm 1.10 \mathrm{D}$, ranging from 0 to $-5.50 \mathrm{D}$; and the cylinder was $-0.95 \pm 0.88 \mathrm{D}$, ranging from -0.00 to $-3.75 \mathrm{D}$. There were significant differences between the high myopia group and the control group on the MRSE and sphere, preoperatively $(\mathrm{p}<0.001$, $\mathrm{p}<0.001$, respectively). There were no statistically significant differences between the high myopic group and the control group in regard to the patients' gender, age, or cylindrical dioptre, preoperatively $(\mathrm{p}=0.835, \mathrm{p}=0.055$, $\mathrm{p}=0.341$, respectively).

\section{Surgical technique}

An experienced surgeon (YW) performed all the SMILE procedures. The Visumax femtosecond laser system
(Carl Zeiss Meditec AG) with a repletion rate of $500 \mathrm{kHz}$ was used for these procedures. The intended cap thickness was $110 \mu \mathrm{m}$ and the diameter of the optical zone was $6.0-6.5 \mathrm{~mm}$. We slightly decreased the optical zone to save more tissue and also to reduce the risk of the keratoectasia in the highly myopic eyes with thinner corneal thickness. Meanwhile, the low mesopic pupil sizes of the subjects were all smaller than the optical zones. All side-cut angles were $90^{\circ}$. The target refraction was $\pm 0.25 \mathrm{D}$. The surgical procedure was under topical anaesthesia with 2-3 drops oxybuprocaine eye drops for 3 min (Benoxil, Santen, Inc, Japan). A standard eyelid speculum was used to keep the eye open, and the patient's eye was positioned under curved contact glass of the femtosecond laser and fixated on a blinking target. The suction was applied when the centre of the pupil was centred to the contact lenses. The posterior of the refractive lenticule was created from the periphery to the centre of the cornea. The anterior surface of the refractive lenticles was created from the centre to the periphery, and finally the small incision was created at the 12 o'clock position with the cordial length ranging from 2 to $5 \mathrm{~mm}$. After laser treatment, our patented spatula was used to break the remaining tissue bridges and loosen the stromal lenticules, which were then grasped with a pair of forceps and removed. All patients received one drop of topical ofloxacin $0.3 \%$ (Tarivid; Santen, Inc, Osaka, Japan) and one drop of diclofenac (Voltaren Ophtha) at the end of the procedure. After that, topical ofloxacin $0.3 \%$ was applied four times daily for 3 days postoperatively, and $0.1 \%$ fluorometholone (Flumetholon; Santen, Inc, Osaka, Japan) was applied four times a day for 2 weeks and then tapered over 2 months.

\section{Preoperative and postoperative assessments}

The subjects had eye examinations including the manifest refraction, UDVA, CDVA, history of refractive stability, slit-lamp evaluation, dilated fundoscopy and corneal topography using a rotating Scheimpflug Camera (Pentacam HR, Oculus, Wetzlar, Germany), preoperatively. The postoperative follow-up was scheduled at 1 day, 1 week, 1 month, 3 months, 6 months and 12 months, including the manifest refraction, UDVA and CDVA examinations. The UDVA and CDVA were measured using a standard Snellen visual acuity chart. The qualified readings preoperatively and postoperatively were accepted for statistical analysis.

\section{Statistical analysis}

Statistical analysis was performed using the SPSS software (V.20.0, Chicago, USA). The normality of all data samples was checked with the Kolmogorov-Smirnov test. The comparisons between the groups for normally distributed data were made using the Student t-test (twotailed). The Mann-Whitney rank-sum test was used for non-normally distributed data between the two independent groups, and the Kruskal-Wallis test was used to 
determine any significant differences among the nonnormally distributed multiple groups. Differences were considered to be statistically significant at $\mathrm{p}<0.05$.

\section{RESULTS}

\section{Visual recovery}

The visual recovery indicates the changes in the UDVA with time after the SMILE surgery. The UDVA was 0.93 $\pm 0.15,0.99 \pm 0.11,1.03 \pm 0.14,1.04 \pm 0.15,1.01 \pm 0.17$, and $0.99 \pm 0.28$ in the high myopia group at 1 day, 1 week, 1 month, 3 months, 6 months, and 12 months, postoperatively. The UDVA was $0.91 \pm 0.15,1.00 \pm 0.18,1.05$ $\pm 0.15,1.05 \pm 0.15,1.08 \pm 0.21$, and $1.09 \pm 0.18$ in the control group at 1 day, 1 week, 1 month, 3 months, 6 months, and 12 months, respectively. There were statistically significant differences among the UDVAs at 1 day, 1 month, 3 months, 6 months, and 12 months in the high myopic eyes $(p=0.003$, Kruskal-Wallis test). The long-term UDVA (1 year) was significantly better than the result at the early stage ( 1 day) $(\mathrm{p}=0.035$, figure $1 \mathrm{~A})$. In the low to moderate myopic group, the UDVA was also better at 1 year than at 1 day $(p=0.016$, figure $1 B)$.

Figure 1C suggests that although the UDVA showed no statistically significant differences between the high myopic eyes and the control group at 1 day $(\mathrm{p}=0.22$, Mann-Whitney Test $), 1$ week $(\mathrm{p}=0.298), 1$ month $(\mathrm{p}=0.514)$ or 3 months $(\mathrm{p}=0.417)$, the UDVA was significantly lower in the high myopia group than the control group at 6 and 12 months $(\mathrm{p}=0.015, \mathrm{p}=0.021)$.

\section{Refractive stability}

Refractive stability depicts the changes of the MRSE with time after the SMILE surgery. The MRSE was -0.05 $\pm 0.33 \mathrm{D}, \quad-0.05 \pm 0.40 \mathrm{D}, \quad-0.05 \pm 0.27 \mathrm{D}, \quad-0.09 \pm 0.24 \mathrm{D}$, $-0.15 \pm 0.20 \mathrm{D}$, and $-0.21 \pm 0.25 \mathrm{D}$ in the high myopia group at 1 day, 1 week, 1 month, 3 months, 6 months, and 12 months, postoperatively. The MRSE was -0.09 $\pm 0.27 \mathrm{D}, \quad-0.05 \pm 0.23 \mathrm{D}, \quad-0.08 \pm 0.19 \mathrm{D}, \quad-0.08 \pm 0.23 \mathrm{D}$, $-0.09 \pm 0.23 \mathrm{D}$, and $-0.07 \pm 0.21 \mathrm{D}$ in the control group at 1 day, 1 week, 1 month, 3 months, 6 months, and 12 months, respectively. It showed statistically significant differences from 1 day to 1 year in the high myopia group $(\mathrm{p}<0.001$, Kruskal-Wallis test), and the absolute values of the MRSE were significantly higher at 1 year than the values at 1 day, 1 week, or 1 month, (figure 2A, $\mathrm{p}=0.048, \mathrm{p}=0.001, \mathrm{p}=0.012$, respectively). However, the postoperative MRSE showed no differences from 1 day to 1 year in the control group ( $\mathrm{p}=0.612$, Kruskal-Wallis test). It stabilised within 1 day after surgery, as shown in figure 2B.

The MRSE was significantly worse in the high myopia group than the control group at 1 year after the SMILE surgery $(p<0.001$, Mann-Withney $U$ test $)$, as shown in figure $2 \mathrm{C}$. There were no significant differences between
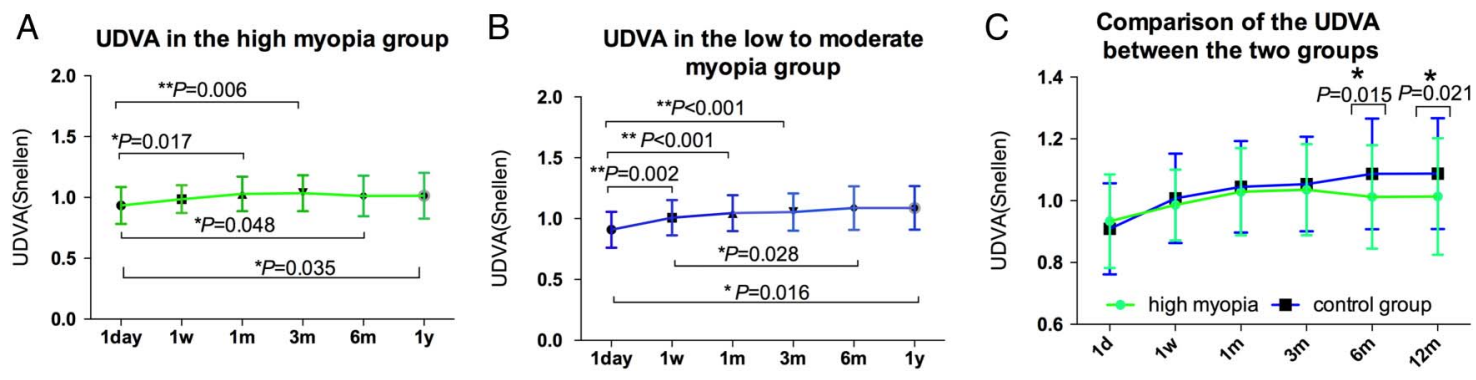

Figure 1 The UDVA in the high myopia group (green, A) and the control group (blue, B) after SMILE surgery, and comparison of the UDVA between the two groups $(\mathrm{C}) .{ }^{*} \mathrm{p}<0.05$, ${ }^{\star *} \mathrm{p}<0.01$. UDVA, uncorrected distance visual acuity; SMILE, small incision lenticule extraction.
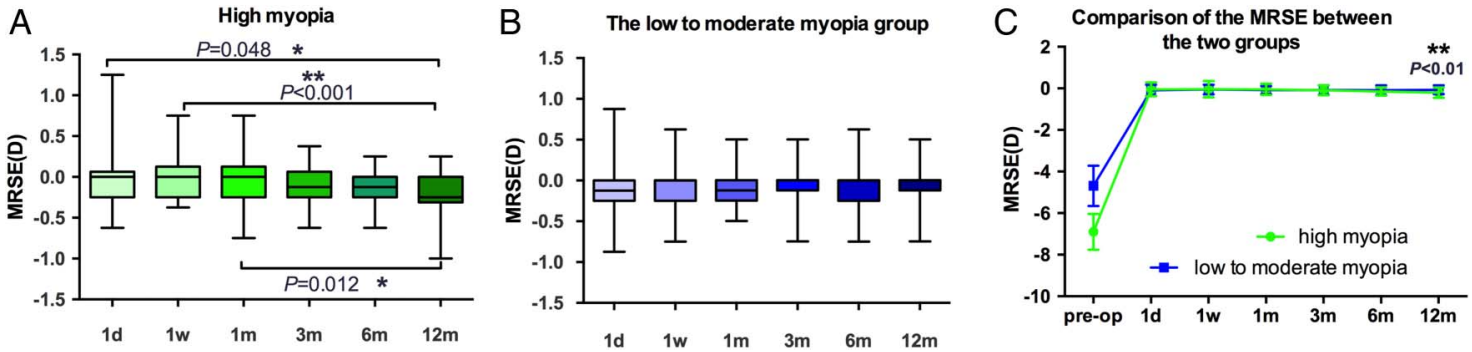

Figure 2 Refractive stability. Plots of the MRSE against time in the high myopia group (green, A), and the control group (blue, $\mathrm{B})$, and comparison of the MRSE between the two groups (C). The height of the box indicates the upper and lower quartiles. The bars in the box indicate the maximum and minimum range of the results; the error bar in the line chart indicates the $S D$. ${ }^{*} p<0.05$, ${ }^{* *} \mathrm{p}<0.01$. MRSE, manifest refraction spherical equivalent. 
Figure 3 The safety index in the high myopia group (green, $A$ ) and the control group (blue, B) at 1 day, 1 week, 1 month, 3, 6 and 12 months after SMILE surgery. The height of the box indicates the upper and lower quartiles. The bars in the box indicate the maximum and minimum range of the results. ${ }^{*} p<0.05 ;{ }^{* *} p<0.01$. SMILE, small incision lenticule extraction.
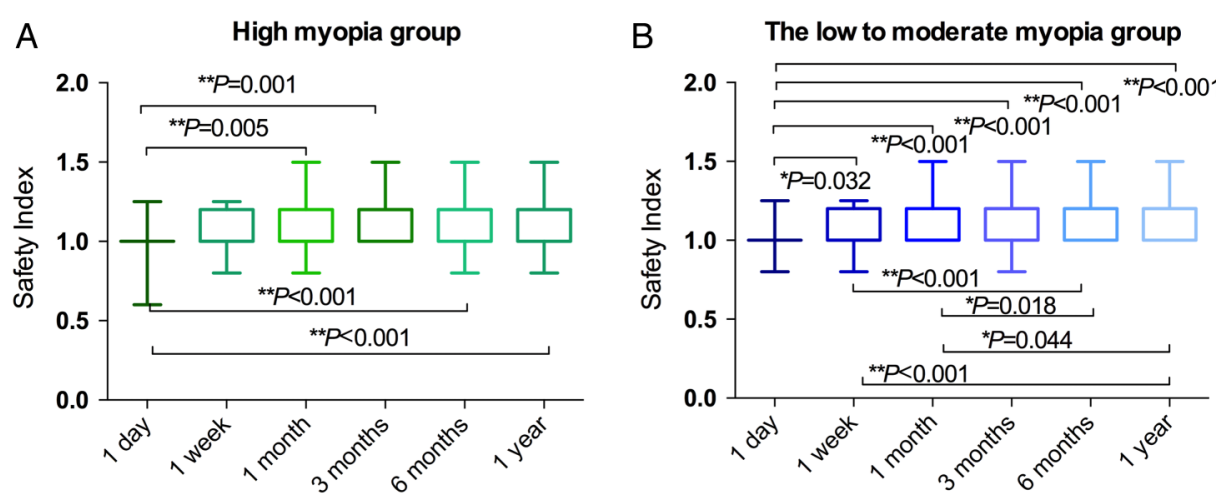

the two groups at 1 day, 1 week, 1 month, 3 months or 6 months $(\mathrm{p}=0.625, \mathrm{p}=0.509, \mathrm{p}=0.433 \mathrm{p}=0.577, \mathrm{p}=0.074$, respectively).

\section{Safety}

The safety index is defined as the postoperative CDVA/ preoperative CDVA. In the high myopia group, the safety index was $1.01 \pm 0.12,1.07 \pm 0.13,1.10 \pm 0.12,1.11$ $\pm 0.11, \quad 1.11 \pm 0.12$, and $1.13 \pm 0.13$ at 1 day, 1 week, 1 month, 3 months, 6 months, and 12 months, postoperatively. In the control group, the safety index was $1.01 \pm 0.11, \quad 1.07 \pm 0.12, \quad 1.10 \pm 0.12, \quad 1.13 \pm 0.14, \quad 1.17 \pm 0.14$, and $1.17 \pm 0.14$ at 1 day, 1 week, 1 month, 3 months, 6 months, and 12 months, respectively. In both groups, there were significant differences in the safety index among the different postoperative times $(p<0.001$, Kruskal-Wallis test), as shown in figure 3. The safety index was significantly better in the long term (6 months and 1 year) after SMILE surgery than at the early stage ( 1 day) in both groups.

There were no significant differences in the safety index between the high myopia group and the control group at 1 day, 1 week, 1 month, 3 months, 6 months or 12 months $\quad(\mathrm{p}=0.645, \mathrm{p}=0.859, \mathrm{p}=0.888, \mathrm{p}=0.512$, $\mathrm{p}=0.091, \mathrm{p}=0.386$, respectively, Mann-Withney U test).

\section{Predictability}

In both groups, the percentages of eyes within $\pm 0.50 \mathrm{D}$ showed no significant difference between 1 day and 1 year follow-up ( $p=1.00, \chi^{2}$ test) (figure 4). Moreover, no significant differences between the two groups were seen at all follow-up visits ( 1 day: $\mathrm{p}=1.000,1$ week: $\mathrm{p}=1.000,1$ month: $\mathrm{p}=0.070,3$ months: $\mathrm{p}=0.641,6$ months: $\mathrm{p}=0.402$, 1 year: $\mathrm{p}=0.694$, Mann-Whitney $\mathrm{U}$ test).

Figure 5 illustrates the regression line within the plot of the actual correction achieved versus the attempted refractive correction 1 year postoperatively. In the high myopia group, the equation for this line could be expressed as follows, $\left(\mathrm{R}^{2}=0.919\right)$,

$$
\begin{aligned}
\text { Achieved SE }(\mathrm{D})= & 0.885 \times \text { Attempted SE }(\mathrm{D}) \\
& +0.587 \mathrm{D}
\end{aligned}
$$

(the absolute value of the SE was used for this equation).
As shown in figure $5 \mathrm{~A}$, it is better to increase the magnitude of the myopic correction for the high myopic eyes to achieve the attempted SE. Based on equation (1), we created a new equation:

$$
\begin{aligned}
\text { Achieved SE }= & 0.885 \times(\text { Attempted SE } \\
& + \text { Adjustment }) \mathrm{D}+0.587 \mathrm{D},
\end{aligned}
$$

(the absolute value of the $\mathrm{SE}$ was used for this equation).

To make the achieved SE equal to the attempted SE, we obtained a new equation,

$$
\begin{aligned}
\text { The adjustment }(\mathrm{D})= & 0.13 \times \operatorname{Attempted} \mathrm{SE}(\mathrm{D}) \\
& -0.66 \mathrm{D}
\end{aligned}
$$
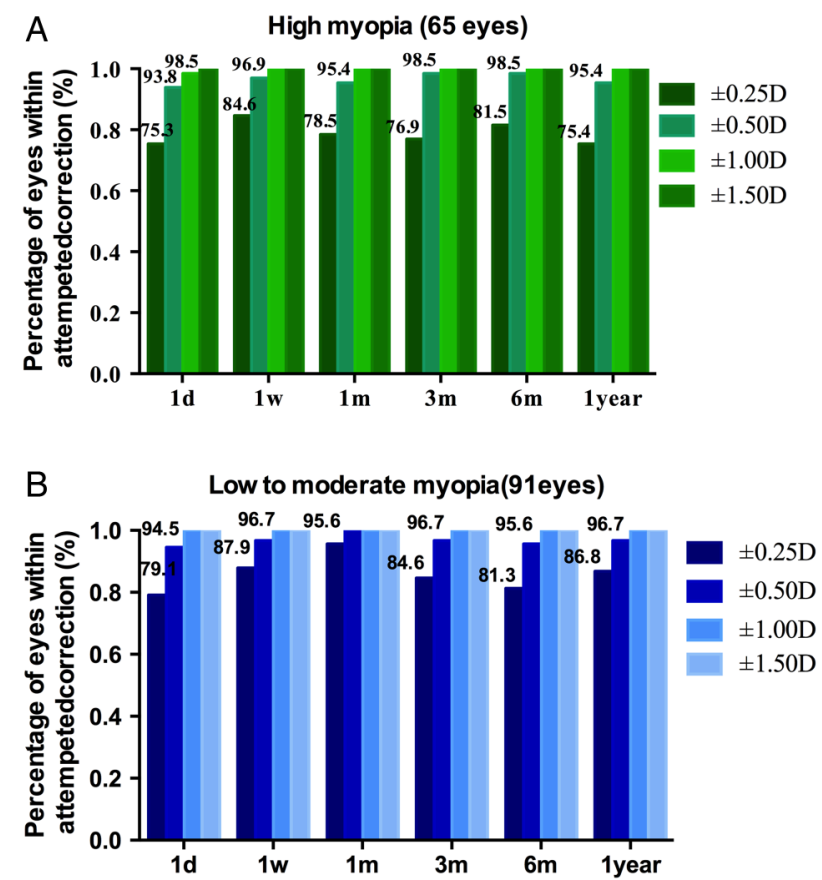

Figure 4 Bar graphs showing the percentage of eyes within $\pm 0.25 \mathrm{D}, \pm 0.05 \mathrm{D}, \pm 1.00 \mathrm{D}$, and $\pm 1.50 \mathrm{D}$ of the target refraction at 1 day, 1 week, 1 month, 3, 6 months and 1 year after SMILE surgery in the high myopic eyes (green bars) and the low to moderate myopic eyes (blue bars). SMILE, small incision lenticule extraction. 
Figure 5 Predictability. Scatter plot of the absolute value of the attempted MRSE changes plotted against the achieved MRSE changes at 1 year in the high myopic eyes (green circles, A) and the low to moderate myopic eyes (blue circles, B). MRSE, manifest refraction spherical equivalent.

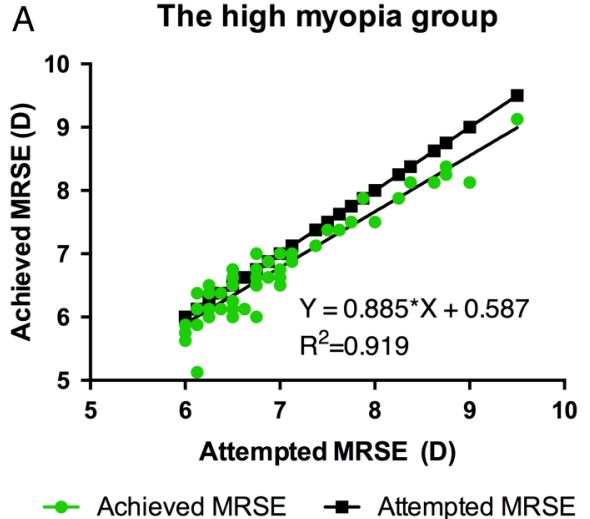

B The low to moderate myopia group

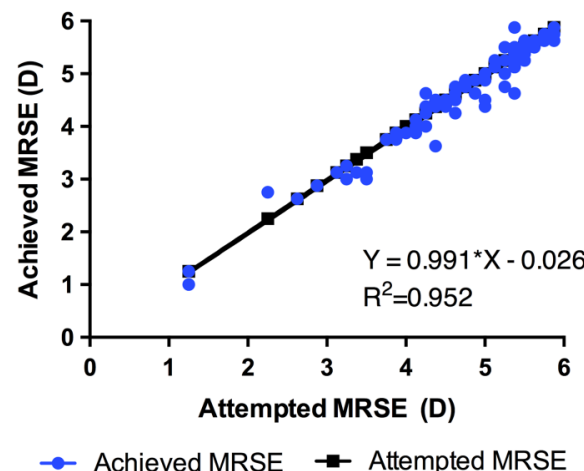

(the absolute value of the SE was used for this equation, the attempted SE ranges from -6.0 to $-9.50 \mathrm{D})$.

For example, if the attempted SE is $-6.50 \mathrm{D}$, according to equation (2), the additional magnitude is $0.13 \times 6.50 \mathrm{D}-0.66 \mathrm{D}=0.185 \mathrm{D}$ (equation (3)); it is better to enter $-6.685 \mathrm{D}(-6.50 \mathrm{D}+(-0.185 \mathrm{D}))$ in the surgical machine to achieve the $-6.5 \mathrm{D}$ correction. This equation may be suitable for the attempted SE ranging from -6.0 to $-9.50 \mathrm{D}$.

The regression line for the low to moderate myopic eyes is expressed using the following equation $\left(\mathrm{R}^{2}=0.952\right)$ :

Achieved SE $(\mathrm{D})=0.991 \times$ Attempted SE $(\mathrm{D})-0.026,(4)$

(the absolute value of the SE was used for this equation).

As shown in figure $5 \mathrm{~B}$, the achieved $\mathrm{SE}$ is well in line with the attempted SE, and no adjustment is needed for the control group.

\section{Complications}

Neither group had visually threatening complications. No cases of epithelial ingrowth, severe diffuse lamellar keratitis, or keratoectasia were seen at any time during the 1-year observation period.

\section{DISCUSSION}

In order to find the long-term (1-year) and short-term visual differences of the SMILE surgery in the high myopic eyes, the present study not only investigated the refractive stability from 1 day to 1 year, but also studied the visual recovery, safety and predictability. Moreover, this study analysed the high myopic eyes separately and showed that the SMILE surgery could provide as safe an outcome for the high myopic eyes as for the low to moderate myopic eyes, even at 1 year postoperatively. This may be clinically important.

The long-term visual acuity and safety results were better than the short-term results in the high myopic eyes following SMILE surgery. This is consistent with published data, showing a continuing improvement of CDVA and safety results after the SMILE surgery. ${ }^{18}$
However, it is still important to detect a keratoconussuspect cornea or a subclinical keratoconus/forme fruste keratoconus as a contraindication to the SMILE surgery to allay any concerns over safety. ${ }^{19-21}$

In terms of the MRSE, the high myopic eyes showed a significant regression over the first year from $-0.05 \mathrm{D}$ at 1 day to $-0.21 \mathrm{D}$ at 1 year $(\mathrm{p}=0.048)$. In contrast, the low to moderate eyes showed no significant changes. The regression in the high myopic eyes may be due to multiple factors, and one of the potential mechanisms may be the relatively more severe corneal wound healing responses that occur in the low to moderate myopic eyes. $^{22}$ Greater keratocyte activation was seen in the high myopic corrections after the SMILE surgery. ${ }^{23}$ These cells may transform to myofibroblast cells and contract during the wound healing process and contribute to the steepening of the cornea. ${ }^{22} 24$ Another possible reason for the regression may be the epithelial hyperplasia, which also correlated positively with the degree of myopia. ${ }^{25}$ Ganesh et al found a significant epithelial thickness increase in the central and superior zones in the high myopic eyes. The epithelial thickness profile changes after SMILE may cause the steepening of the cornea and the regression. $^{25}$ The small optical zone, weak corneal biomechanics, low preoperative pachymetry and low residual stromal bed may also be predictors of myopic regression in the high myopic eyes. ${ }^{18} 27-31$

In addition, Blum et al found the regression was $-0.48 \mathrm{D}$ over a 5-year follow-up after SMILE surgery; they assumed this might be due to an increase of the axial length as a consequence of eye globe growth rather than a true regression at the corneal level. ${ }^{32}$ Hence, further investigations on regression following SMILE surgery are required. ${ }^{18}$

In terms of predictability, the short-term predictability is similar to the long-term results obtained both in the high myopic eyes and the low to moderate myopic eyes. Sekundo et $a l^{13}$ reported $92 \%$ of eyes were within $\pm 0.50 \mathrm{D}$ at 12 months after the SMILE surgeries, and in a study by Agca et al this figure was 95\%..$^{5}$ Recently, Kim et al reported $88 \%$ of the high myopic eyes and $87.9 \%$ of the low to moderate myopic eyes were within $\pm 0.50 \mathrm{D}$ 1 year after SMILE surgery. ${ }^{17}$ Moshirfar et al also 
suggested that SMILE surgery could offer good predictability and safety. ${ }^{24}$ Our results are comparable with these previously published studies, and these investigations together suggest that the SMILE procedure shows high predictability in both groups, even in the long term.

There are some limitations in this study. This is a retrospective cohort study, and is potentially prone to selection bias. To reduce this, we compared the differences between the high myopic eyes and the control group; there were no significant differences in the patients' gender, age, or cylindrical dioptre, preoperatively. The authenticity of the results would probably have been better confirmed in a randomised controlled study with a large sample. Future studies are also needed to determine the differences in the corneal biomechanics, and the high order aberrations. As the optical zone varied with the degree of the myopia and the corneal thickness, it may limit our future studies on corneal biomechanics and high order aberrations.

In summary, the long-term (1-year) results were found to be better than the short-term results following SMILE surgery in terms of visual acuity and safety. The 1-year predictability remained similar with the early stage. However, the high myopic eyes suffered a significant regression over the first year, which may be corrected by adding additional magnitude to the SE.

Acknowledgements We would like to give special thanks to Mohamed Abdelwahed for the language editing.

Contributors WWJ performed, designed the study, and drafted the manuscript. ZH, ZJM, LH and DR conducted the long-term observation and participated in the examination and acquisition of data. WY conceived the study, participated in the design, revised the manuscript and gave final approval of the version for publication. All authors read and approved the final manuscript.

Funding This study was supported by research grants from the National Nature Science Foundation of China (Grant No. 81470658. to Y.W.) and Tianjin Research Program of Application Foundation and Advanced Technology (14JCZDJC35900).

Competing interests None declared.

Patient consent Obtained.

Ethics approval The Institutional Review Board of Tianjin Eye Hospital.

Provenance and peer review Not commissioned; externally peer reviewed.

Data sharing statement No additional data are available.

Open Access This is an Open Access article distributed in accordance with the Creative Commons Attribution Non Commercial (CC BY-NC 4.0) license, which permits others to distribute, remix, adapt, build upon this work noncommercially, and license their derivative works on different terms, provided the original work is properly cited and the use is non-commercial. See: http:// creativecommons.org/licenses/by-nc/4.0/

\section{REFERENCES}

1. Sekundo $\mathrm{W}$, Kunert KS, Blum M. Small incision corneal refractive surgery using the small incision lenticule extraction (SMILE) procedure for the correction of myopia and myopic astigmatism: results of a 6 month prospective study. $\mathrm{Br} J$ Ophthalmol 2011;95:335-9.
2. Shah R, Shah S, Sengupta S. Results of small incision lenticule extraction: all-in-one femtosecond laser refractive surgery. J Cataract Refract Surg 2011;37:127-37.

3. Hjortdal JØ, Vestergaard AH, Ivarsen A, et al. Predictors for the outcome of small-incision lenticule extraction for myopia. J Refract Surg 2012;28:865-71.

4. Vestergaard A, Ivarsen AR, Asp S, et al. Small-incision lenticule extraction for moderate to high myopia: predictability, safety, and patient satisfaction. J Cataract Refract Surg 2012;38:2003-10.

5. Ağca A, Demirok A, Cankaya Ki, et al. Comparison of visual acuity and higher-order aberrations after femtosecond lenticule extraction and small-incision lenticule extraction. Cont Lens Anterior Eye 2014;37:292-6.

6. Ang M, Mehta JS, Chan C, et al. Refractive lenticule extraction: transition and comparison of 3 surgical techniques. J Cataract Refract Surg 2014;40:1415-24.

7. Ganesh S, Gupta R. Comparison of visual and refractive outcomes following femtosecond laser- assisted Lasik with smile in patients with myopia or myopic astigmatism. J Refract Surg 2014;30:590-6.

8. Ivarsen A, Asp S, Hjortdal J. Safety and complications of more than 1500 small-incision lenticule extraction procedures. Ophthalmology 2014;121:822-8.

9. Ivarsen A, Hjortdal J. Correction of myopic astigmatism with small incision lenticule extraction. J Refract Surg 2014;30:240-7.

10. Kamiya K, Shimizu K, Igarashi A, et al. Visual and refractive outcomes of femtosecond lenticule extraction and small-incision lenticule extraction for myopia. Am J Ophthalmol 2014;157:128-34 e122.

11. Alió JL, Muftuoglu O, Ortiz D, et al. Ten-year follow-up of photorefractive keratectomy for myopia of more than -6 diopters. Am J Ophthalmol 2008;145:37-45.

12. Alió JL, Muftuoglu O, Ortiz D, et al. Ten-year follow-up of photorefractive keratectomy for myopia of less than -6 diopters. Am J Ophthalmol 2008;145:29-36.

13. Sekundo W, Gertnere J, Bertelmann T, et al. One-year refractive results, contrast sensitivity, high-order aberrations and complications after myopic small-incision lenticule extraction (ReLEx SMILE). Graefes Arch Clin Exp Ophthalmol 2014;252:837-43.

14. $\mathrm{Xu} \mathrm{Y,} \mathrm{Yang} \mathrm{Y.} \mathrm{Small-incision} \mathrm{lenticule} \mathrm{extraction} \mathrm{for} \mathrm{myopia:}$ results of a 12-month prospective study. Optom Vis $\mathrm{Scl}$ 2015;92:123-31.

15. Zhang J, Wang $\mathrm{Y}, \mathrm{Wu} \mathrm{W}$, et al. Vector analysis of low to moderate astigmatism with small incision lenticule extraction (SMILE): results of a 1-year follow-up. BMC Ophthalmol 2015;15:8

16. Pedersen IB, Ivarsen A, Hjortdal J. Three-year results of small incision lenticule extraction for high myopia: refractive outcomes and aberrations. J Refract Surg 2015;31:719-24.

17. Kim JR, Kim BK, Mun SJ, et al. One-year outcomes of small-incision lenticule extraction (SMILE): mild to moderate myopia vs. high myopia. BMC Ophthalmol 2015;15:59.

18. Moshirfar M, McCaughey MV, Reinstein DZ, et al. Small-incision lenticule extraction. J Cataract Refract Surg 2015;41:652-65.

19. El-Naggar MT. Bilateral ectasia after femtosecond laser-assisted small-incision lenticule extraction. J Cataract Refract Surg 2015;41:884-8.

20. Wang Y, Cui C, Li Z, et al. Corneal ectasia 6.5 months after small-incision lenticule extraction. J Cataract Refract Surg 2015;41:1100-6.

21. Mastropasqua L. Bilateral ectasia after femtosecond laser-assisted small-incision lenticule extraction. J Cataract Refract Surg 2015;41:1338-9.

22. Wilson SE. Analysis of the keratocyte apoptosis, keratocyte proliferation, and myofibroblast transformation responses after photorefractive keratectomy and laser in situ keratomileusis. Trans Am Ophthalmol Soc 2002;100:411-33.

23. Liu YC, Teo EP, Lwin NC, et al. Early corneal wound healing and inflammatory responses after SMILE: comparison of the effects of different refractive corrections and surgical experiences. $J$ Refract Surg 2016;32:346-53.

24. Moshirfar M, Anderson E, Hsu M, et al. Comparing the rate of regression after conductive keratoplasty with or without prior laser-assisted in situ keratomileusis or photorefractive keratectomy. Middle East Afr J Ophthalmol 2012;19:377-81.

25. Ganesh S, Brar S, Relekar KJ. Epithelial thickness profile changes following small incision refractive lenticule extraction (SMILE) for myopia and myopic astigmatism. J Refract Surg 2016;32:473-82.

26. Lohmann CP, Reischl U, Marshall J. Regression and epithelial hyperplasia after myopic photorefractive keratectomy in a human cornea. J Cataract Refract Surg 1999;25:712-15. 
27. Rajan MS, O'Brart D, Jaycock $\mathrm{P}$, et al. Effects of ablation diameter on long-term refractive stability and corneal transparency after photorefractive keratectomy. Ophthalmology 2006;113:1798-806.

28. Alió JL, Soria F, Abbouda A, et al. Laser in situ keratomileusis for -6.00 to -18.00 diopters of myopia and up to -5.00 diopters of astigmatism: 15-year follow-up. J Cataract Refract Surg 2015;41:33-40.

29. Shojaei A, Mohammad-Rabei H, Eslani M, et al. Long-term evaluation of complications and results of photorefractive keratectomy in myopia: an 8-year follow-up. Cornea 2009;28:304-10.

30. Binder PS. Analysis of ectasia after laser in situ keratomileusis: risk factors. J Cataract Refract Surg 2007:33:1530-8.

31. Sinha Roy A, Dupps WJ Jr, Roberts CJ. Comparison of

biomechanical effects of small-incision lenticule extraction and laser in situ keratomileusis: finite-element analysis. $J$ Cataract Refract Surg 2014;40:971-80.

32. Blum M, Täubig K, Gruhn C, et al. Five-year results of small incision lenticule extraction (ReLEx SMILE). Br J Ophthalmol 2016;100:1192-5. 